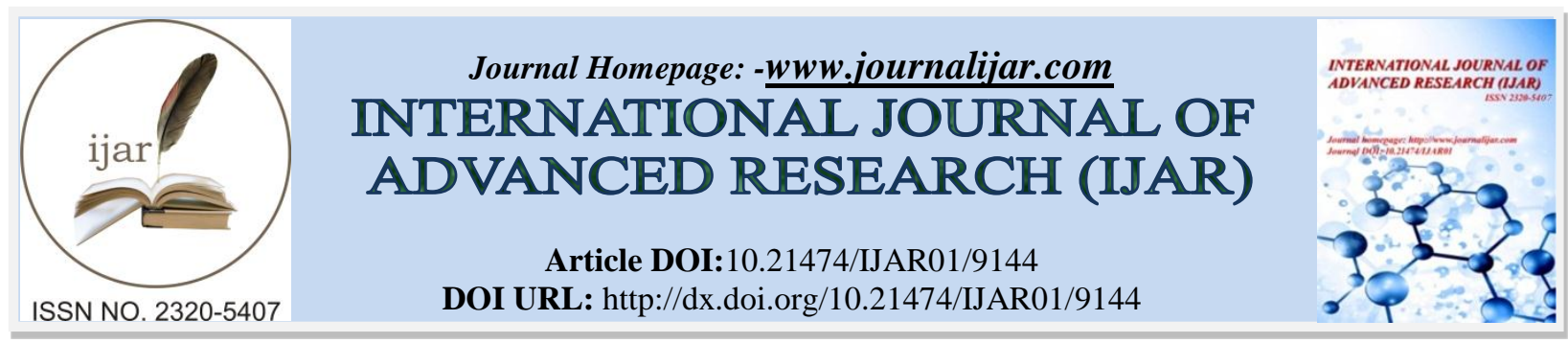

RESEARCH ARTICLE

\title{
ROLE OF MASSIVE OPEN ONLINE COURSES IN INDIAN EDUCATION SYSTEM.
}

\author{
Dr. Sonal Pathak. \\ Associate Professor, Faculty of Computer Applications Manav Rachna Internatonal Institute of Research and \\ Studies, Faridabad.
}

\section{Manuscript Info}

\section{Manuscript History}

Received: 24 March 2019

Final Accepted: 26 April 2019

Published: May 2019

Key words:-

distance education, MOOC Platform, massive open online courses, MOOCs, digital education.

\begin{abstract}
Massive Open Online Courses (MOOCs) have emerged as one of the most potential tool to support quality education and provide training to huge domain of audience worldwide. It reaches to mass of learners, scholars, scientists, professors, teachers and different stakeholders related to education. Promulgate knowledge is available on an efficient digital platform, apart from interlinking a huge network of students. In this paper, features of MOOC courses have been analyzed. Competitiveness of MOOC platforms is studied and quality of content and instructional design issues has been discussed. Some key issues related to MOOCs considering Higher Education system and its utilization by potential education market of India have been discussed. The author has also tried to suggest several possible way outs to implement in virtual learning environment pertaining to an Indian perspective. This article will give a necessary overview of what MOOCs are, how they execute, and some of their advantages and limitations.
\end{abstract}

Copy Right, IJAR, 2019,. All rights reserved.

\section{Introduction:-}

Massive Open Online Courses (MOOCs) are innovative online classes that allow anywhere, anyone, to learn via discussion forums, video lectures and computer graded tests. It helps in exploration about various courses available on MOOC's platforms. MOOC $\mathrm{s}$ libraries includes a list of MOOC related resources and all available courses. India after US is dominating the global growth in enrolments in these courses. Seeing the growth of enrolment from the country and satisfy their need of education, India has started various projects for offering MOOC courses. Currently, NPTEL, MooKIT, IITBX and SWAYAM are the platforms used in India for offering courses. In this paper, a theoretical and technical background of these platforms is provided with a discussion of their features. Besides viewing MOOCs as a route to ubiquitous learning. Various Index terms - MOOCs, ubiquitous learning, distance education, E-learning, virtual classroom, digital divide, digital literacy are used to distinguish between terms. Despite intensified advantages and efficiency, people accessing MOOCs from the developing countries (especially India) do not count to a significant number. India being a promising abode of education too offers a prospective area to MOOCs for large scale implementation. But several factors and constraints like low digital literacy and lack of massive digital infrastructure hinder this process of extensive implementation of MOOCs. Online learning uses technology for delivering the courses. Education with technology is considered as most promising development in education. With technology globalization, the concept of learning and teaching has undergone a tremendous change. Technological usage in education provides global learning environment, which

Corresponding Author:-Dr. Sonal Pathak.

Address:-Associate Professor, Faculty of Computer Applications Manav Rachna Internatonal Institute of Research and Studies. Faridahad 
allows accessing the course material anytime, anywhere, connect other learners, and get access to the content without considering any geographical boundaries. The significant changes in use of the technology in online education has seen emergence of the concept of Massive Open Online Course (MOOC). Even after all the prevalent constraints, India leads the developing nations in terms of MOOCs enrollment. The $21^{\text {st }}$ century witnessed an educational paradigm shift, stemming from the widespread use of Information and Communication Technologies (ICT). With the proliferation of ICT, online, open, and flexible learning moved from the periphery to mainstream education. ICT improved the quality and capacity of the online delivery of educational content. Online networks are used as learning spaces that are distributed, flexible, accessible, and, most importantly, potentially open. Openness in education has evolved over time and has emerged in different forms (Weller, 2014)[15]: It is suggested that "there are three key strands that lead to the current set of open education core concepts: open access education, open source software and web 2.0 culture, providing access to higher learning opportunities is the raison d'être of Open Universities (Tait, 2008)[7]. Massive Open Online Courses (MOOCs) are a recent development of this open learning movement, which have drawn much attention from both the academic and the public sphere. The first course in this format was offered in 2008 at the University of Manitoba and was entitled Connectivism and Connective Knowledge (Liyanagunawardena, Adams, \& Williams, 2013)[8].

\section{History of Emergence}

Prior to the flood of digital era, distance education was mostly existent in the forms of correspondence courses. During the years of 1920-1922, distance education was becoming more and more popular within the mass. Though the primary medium of distance education was based on postal service, with the radio and television getting popular, they were used as the chief mediums of distance education. However, distance education over radio and television were having different flaws and limitations. As the digital age was creeping in, video lectures were used to train a mass of viewers, though in sparse. Not all of them were formal students, neither the training was of some formal education; still the seed of MOOCs was sown in this period only. By 1980s, a remote classroom system was introduced based on close-circuit televisions. This was also another approach of video-based remote classrooms imparting training virtually to a huge audience. With the approach of internet, the term "online" actually came into working. After 1990s, this method of teaching online in a huge domain of learners gradually started to become popular, though in a very slow stride. After the year 2000, an initiative was slowly taken across the world to curb the commoditization of knowledge and knowledge-resources, as UNESCO designates, "teaching, learning and research materials in any medium, digital or otherwise, that reside in the public domain or have been released under an open license that permits no-cost access, use, adaptation and redistribution by others with no or limited restrictions." However, the open licensing was so designed to comply with the author's intellectual property rights and other copyrights. This paradigm of Open Educational Resources (OER) was also helpful in making the study materials more accessible through digital methods. MOOCs emerged from this OER movement, in the hands of Dave Cormier of the University of Prince Edward Island and senior research fellow Bryan Alexander of the National Institute for Technology in Liberal Education, as they coined the term in 2008. The context was a course called "Connectivism and Connective Knowledge", whose audience was a 2200-head general public [3].

\section{Status of MOOC in India}

At its inception, MOOCs was mostly considered as a medium to enable people gain basic education and some workplace skills. But with the introduction of online interaction facilities and increasing domain of stakeholders, it became more and more popular across globe. In India interest shown by aspirant is significant.

Figure1:-Worldwide Interest on MOOCs (Jan 2012 - Feb 2014)

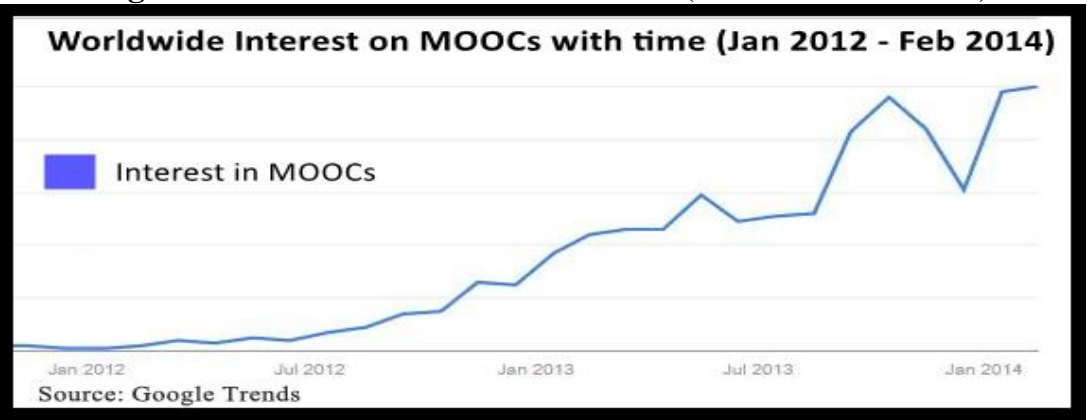


A survey report (Fig. 1) shows that the interest for MOOCs has increased considerably with time in a worldwide domain. Google power search results for the keywords (MOOC, OCWs etc.) for google's complete database (world) revealed that online course ranked first (37.22\%) followed by OCWs (21.57\%), online education (16.36\%), MOOC $(16.05 \%)$ and video lectures $(8.8 \%)$. This shows that the documents available on the internet are largely dominated by online course(s) keyword which is a very generic term for ICT enabled education. Table 1 has been showing some Indian MOOC platforms and providers.

Table I:-Some Indian MOOC Platforms and Providers

\begin{tabular}{|l|l|l|l|}
\hline Initiative & Year of Launch & Institution Behind Platform & Website Link \\
\hline NPTEL & 2003 & IIT Madras & nptel.ac.in/ \\
\hline mooKIT & 2012 & IIT Kanpur & www.mookit.co/ \\
\hline IITBX & 2014 & IIT Bombay & iitbombayx.in/ \\
\hline SWAYAM & 2016 & MHRD and Microsoft & swayam.gov.in \\
\hline
\end{tabular}

\section{Challenges For MOOC in India}

Some of the major concerns regarding the implementation of MOOCs in India are, the lack of technological infrastructure, investment, diversified population, quality of courses, adoption of MOOC among learners and their acceptance by the academic institutions:

\section{Technological Infrastructure:}

MOOC needs the high speed internet connections for accessing the content delivered in their courses. In a developing country like India, Internet and computers comes under luxury and their availability is confined mainly to the urban areas. Limited availability of requisite infrastructure to access MOOCs has confined the extensive spread of MOOCs [9]. India needs to work towards providing a better Internet access for the country's population and provides worldwide connectivity.

\section{Investment:}

Offering the MOOC is a costly affair that includes the cost of infrastructure, platforms, content creation, human resources and many more. In India, the institutions do not have many capitals to invest in such event so it is not easy for an individual institution to offer such services. There is need of involvement from some authorities, who can invest in the process to support the education. Even Indian government needs to liberalize conventional regulations and restrictions and encourage public private partnership for creating MOOCs in this country [10].

\section{Diversified Needs:}

India is a widely diversified country having multicultural societies and different languages spoken. For acceptance of MOOC among the huge domain of audience, they need to agree upon a common language of speaking. English as this language accepted globally, again throws away a considerable amount of audience who do not possess the knowledge or adequate fluency in English [17]. So, a switch over to mostly English based courses as offered via current MOOCs often discourages learner to continue their courses.

The courses should also be offered in some regional languages, which may be a tedious task and prone to loss of uniformity and quality. Therefore, language is one of the barriers for learners from Indian origin that need to be addressed by the MOOC providers in a more realistic way. Moreover, the challenge is to deliver the lecture, designing of the course material, and the platform itself, in a way that can be understood by all. Hence forth, the main motive should be to work in the direction which can minimize the existing differences amongst the learners [10].

\section{Adoption of MOOC among learners:}

Generally in MOOC courses the communication between a teacher and learner and among learner as well is in written form. It results in the lack of oral communication skills among the learners and to improve this they need undergo a traditional program. Also, watching the course videos of other content on a computer screen can make the learner feel isolated. Due to this, motivation of learner falls resulting in dropping out from the course. Moreover, the courses that require lab or hands-on training may not fulfill the purpose completely in online mode [10]. Therefore, the challenge faced by MOOC could be adoption of technology by learners. 


\section{Quality:}

To create and deliver quality content in MOOC, quality of teachers and technical staff is required. India has huge vacancies of teachers not filled, compounded by infrastructure deficit as the absence of laboratories. Also, it may be possible that teachers are not technically sound to create course content using the tools. Emerging initiatives internationally and nationally are working towards offering quality educational by providing their content as open resources, but some of them are constraint by the adoption policies of their country or organization. India should also need to leverage these initiatives as a readily available, economically viable source of quality content for adoption [11].

Also, a national quality assessment framework to assess the quality and adoption of new approaches like, credit transfer, MOOC, integrated courses etc., should be adopted along with teacher training, their performance related appraisal and midterm re-evaluation [12].

Thus a complete revamp is needed to meet the present demand and address the challenges that India is facing in offering MOOCs.

\section{Factors For Implementing MOOCs in India}

Across India, as in many other contexts, cascade models are the dominant mechanism for reaching large numbers of professionals. MOOC -India aimed to disrupt this approach; cascade models have limited efficacy and perpetuate hierarchies of professionals with front-line workers - teachers and teacher educators, receiving training after mediation by those more distant from the point at which the problem is greatest (Elmore, 1980)[5]. A MOOC, with its ability to enroll concurrently very large numbers of learners, has the potential to make available access to equitable learning experiences for participants across systems and locations. It opens the possibility for participants to exercise agency with respect to their professional learning (Edwards, 2015)[6], negotiating their own learning journey to meet their specific professional needs within the assessment framework. However in designing and delivering the India MOOC there was few prior experiences of similar MOOCS to draw on, although MOOCs are not new to India. Indeed large numbers of Indian participants have engaged with MOOC offerings from global platforms, and an emerging set of local MOOCs is being deployed to support professional training (Alcorn, Christensen, and Kapur 2015)[2]. But evidence suggests participants in these MOOCs are very different to the TESS-India target group; a large proportion of Indian MOOC participants are students enrolled in traditional university program that use MOOC study to augment their campus experience. Alcorn and colleagues (2015, pp. 4647 ) describe Indian MOOC students as predominately male ( 80\%), average age 26 , working in the IT, business or management sectors (58\%) and based in the largest cities. To date there is little evidence of MOOC participation outside the large urban centers and from those engaged in 'front line' activities in sectors such as education and health. A rare exception is the horticultural MOOC from the Commonwealth of Learning delivered on mobile phones to gardeners and agricultural students in Uttar Pradesh (Yadav, 2015). Facilitators were often undertaking this role for the first time and many took personal pride in supporting completion in their group, contacting participants individually and posting updates and photographs online following each class. Analysis of the English MOOC survey responses show weak to medium correlations between overall satisfaction with the MOOC and a range of indicators relating to in-class facilitator support, receiving helpful feedback and the value of attending classes for motivation and discussing online content with others. But the financial and time costs for travel to the contact classes limited attendance for some participants and caused them to withdraw.

A participant also supports each other through MOOC forums and assignment feedback. The post-course analysis of MOOC courses shows that most participants who completed the MOOC had previous experience of providing peer feedback (86.2\%) although only $14.7 \%$ did this 'quite often.' Almost all respondents (96.3\%) said that during the MOOC they provided peer feedback at least once, which means that for around $10 \%$ of participants, the MOOC may have provided their first opportunity in delivering peer feedback to colleagues. This is not to say, however, that participants did not find the activity challenging: $23.4 \%$ admitted to finding it 'very challenging' and $39.3 \%$ found it 'challenging.' No statistically significant differences at the $\mathrm{p}=.05$ level were found between men and women in respect to their participation in providing peer feedback during the MOOC or the degree of challenge this presented. On occasion, participants were disappointed by the brevity of the peer feedback they received, despite guidance being provided and an unanticipated challenge arose when participants received peer feedback in an unfamiliar language. During the MOOC delivery a third strand of participant generated support emerged. This took multiple forms, for example in Assam a DIET Principal recruited a 'computer friendly person' from the community to provide support for himself and members of his staff. In several states facilitators and / or participants utilized social media, creating Whatsapp, Facebook, Share-it and Google groups. Many of these groups were 'invisible' to the 
project team but we are aware of over a dozen Whats App groups in each MOOC iteration, in which participants reminded each other of deadlines, class times, shared ideas and asked for advice. Typically, comments focused on giving procedural information on the classes or giving encouragement to participants.

\section{A Way to Ubiquitous Learning}

The MOOC in India offered an innovative way to support professional development within a large-scale change project, disrupting traditional cascade models. Utilizing a MOOC, in this way for learning specific professional skills promoted a democratic approach in which all participants have space to contribute, congruent with the pedagogies advocated in policy. It developed a collective sense of shared endeavor for participants and was highly cost efficient, particularly when available in the dominant language. Research identifies key constraints on MOOC uptake and completion in developing countries as including awareness of MOOCs, access to IT infrastructure, relevance to country context, and perception of personal value and personal factors (Alcorn et al, 2015; Pompe, nd) [1]. These were relevant here but we suggest 'support for study' is also critical. Success can be judge - in terms of participant completion and changes in subsequent classroom teaching - to a blend of the digital and physical learning spaces, which help collapse the global into the local.

Our findings challenge previous research (Milligan \& Littlejohn, 2014)[13], which found little transfer of learning to on-the job practices for health professionals participating in MOOCs. Project designed attributes such as the authenticity of the study and assessment tasks, the provision of contact classes with trained facilitators and consideration of technology access, combined with local adaptations initiated by participants to meet particular contextual needs. Imaginative user owned solutions were found to overcome infrastructure and access challenges and tools, such as social media harnessed by participants in self-generated support groups. Through the combination of the MOOC platform, contact classes and social media, the MOOC bridged local and distributed learning, creating a hybrid space focused on a shared 'domain of practice' (Wenger, 1998)[16] in which participants could begin to 'learn to be' (Brown, 2005)[2]. There has been much skepticism about the usefulness of MOOCs to support sustainable development. The relative success of the MOOC in India indicates contextualized or localized MOOCs have potential in less economically developed contexts to target skills and capabilities of specific groups of professionals. But success demands careful attention to the provision of an enabling structure that opens possibilities for participants to generate their own solutions to issues of access and support alongside what was formerly provided. Creating such spaces for experimentation and innovation is not always easy, particularly in a context where historically flows of information and learner activity have been highly monitored and controlled.

\section{Proposed Ways to Boost the Spread of MOOCS in INDIA}

Those participants who have completed the MOOC reported overwhelmingly positive experiences, for example, $73 \%$ described their overall MOOC experience as very good and $23 \%$ as good. This means that that $96 \%$ and $98 \%$, respectively rated their MOOC experience as either very good or good. A high percentage $(96 \%)$ of respondents reported that MOOC courses were useful whilst agreed somewhat that it had been useful in their professional role. Also, for almost all participants, the MOOCs helped to improve the effectiveness of teaching (98\%) and the use of a more learner-centered pedagogy in their teaching (98\%). One particularly strong correlation was between satisfaction with the MOOC and usefulness for professional learning. Project reports substantiate this, "participants are finding the course interesting and meaningful to their own situation"' (Assam MOOC Report) [4]. Just under half of those who responded to the pre-course survey listed gaining a Certification of Completion as a 'main reason' for taking part. Historical ways of recognizing learning within a highly hierarchical system may have accorded value to the Certificate of Completion.

A theme through all participant feedback was an appreciation of the value of exchanging ideas with their peers; this practice was new for many participants and was reported to enhance understanding of the OER and participatory pedagogy. The MOOC attempted to position all participants as having something of value to contribute and as one participant commented, "the MOOC has promoted peer learning and support without any competition and encourages collaborative learning". Movement towards more participatory ways of teaching and learning is ongoing and long term but there is evidence that the MOOC experience began to challenge participants' previous views that teacher-created material is always of higher value than peer, or student, generated contributions and that learning is restricted to knowledge of specific facts. Whilst the MOOC India focus was on project implementation in India, the open global nature of the MOOC was important; participants reported appreciating reading different points of view from peers in other countries. Following completion of the MOOC courses, the post-course survey responses indicated that approximately half of the respondents had used the Key Resources. Reported changes in 
practice included. Students are now eager to do things on their own and have started coming every day. An important learning outcome for participants was developing competence and confidence in using technology. This MOOC has eradicated their myth of not being able to pursue and complete an online course'. Group teaching in front of remote TV screens? This is not only an awful way to undertake distance learning, but flies in the face of everything that we have learned while conducting successful open and supported learning on a massive scale for the past 27 years. Our lessons are the key to addressing the triple crisis of access, cost and flexibility now facing higher education world-wide. The synchronous mode of delivery in particular should be improvised which limits access and flexibility, and video-conferencing as a very teacher- centered form of instruction should also be improved . Given the huge demand for open access courses, flexibility, and costs in higher education are obviously still relevant. Furthermore, the evaluation of MOOCs and quality assurance is a very prominent and relevant topic in the publications. Rather than developing new quality frameworks for MOOCs from scratch, it is recommended to build upon quality models and instruments that were developed to measure the quality of multimedia applications, learning objects, and open educational resources (see Yuan \& Recker, 2015).[14]

After this first wave of MOOC hysteria, research and practice should focus on how best to harness the enormous opportunities that MOOCs might afford for providing access to knowledge and education, whilst equally addressing problematic issues like high dropout rates and the development of sustainable cost models. Major lessons learnt from the field of open, distance, and flexible learning (see Zawacki-Richter \& Anderson, 2014)[16], especially in the area of student support, instructional design, and quality assurance, should be kept in mind whilst moving forward.

\section{Conclusion:-}

MOOCs is not only an effective tool to offer quality education in a diversified and open way, but also is a major rising power to compete with the traditional form of regular education in schools, colleges, and universities. Ubiquitous learning is another tool in this aspect which would also work on the same goal in decentralizing the form of education and opening up the domain to a huge global extent. MOOC platforms are being used globally for offering online course and India is no exception. There are various MOOC platforms that are being used in India for offering the courses, such as, NPTEL, mooKIT, IITBX, and SWAYAM. Except the NPTEL, these are the new platforms having history of hardly 4 to 5 years, while SWAYAM is launched very recently. Therefore, to set up a ground for understanding including theoretical as well as technical aspects, a continuous improvisation is required with their advanced features. Also, while using these platforms it is needed to understand their current state, popularity among learners, use of social media for referring, searched etc, and several other parameters or features. For the purpose of assessment of success, web analysis considering several parameters is to be developed and utilized. Furthermore, there are some issues that are faced while implementing MOOC in India. Some of these issues are already addressed by SWAYAM, which is the most recent platform. On one hand MOOCs would bring a huge audience under the light of quality education that was not able to get it due to different constraints fixed up by the academic institutions or some other causes. And on the other hand Ubiquitous learning would provide a global platform of exchange between the different stakeholders of education.

Project designed attributes such as the authenticity of the study and assessment tasks, the provision of contact classes with trained facilitators and consideration of technology access, combined with local adaptations by participants to meet particular contextual needs. Imaginative user owned solutions to overcome infrastructure and access challenges and tools, such as social media harnessed in self-generated support groups. Through the combination of the MOOC platform, contact classes and social media, the MOOC bridged local and distributed learning, creating a hybrid space focused on a shared 'domain of practice' in which participants could begin to 'learn to be'. There has been much skepticism about the usefulness of MOOCs to support sustainable development. The relative success of the India MOOC indicates contextualized or localized MOOCs have potential in less economically developed contexts to target skills and capabilities of specific groups of professionals. But success demands careful attention to the provision of an enabling structure that opens possibilities for participants to generate their own solutions to issues of access and support alongside what was formerly provided. Creating such spaces for experimentation and innovation is not always easy, particularly in a context where historically flows of information and learner activity have been highly monitored and controlled. Basically MOOCs would promote Ubiquitous Learning, transforming the educational epicenter from blackboard to keyboard. As a result the total educational quality would see a massive uplift as a whole. 


\section{References:-}

1. Aspiring Minds (2016), National Employability Report 2016 - Aspiring Minds, Annual Report.

2. Boyd, G., \&Kasraie, N. (2013). Can MOOC fires bring light to shadow education? International Journal of Learning and Development, 3(4),87-95.

3. Bhattacharyya, R. (2013, July 19). Seven IITs, Infosys, TCS, Cognizant and Nasscom team upto provide free online courses. The Economic Times [New Delhi]. Retrieved fromhttp://articles.economictimes.indiatimes.com /2013-07-19/news/40681617_1_seven-olderiits-nptel-andrew-thangaraj.

4. Clifton, B. (2012). Advanced web metrics with Google Analytics. John Wiley \& Sons.

5. Damodharan, D. (2016, Jul 15). India Represents The Second Highest Enrolments in Our Courses': EdX CEO Anant Agarwal, Blog. http://www.huffingtonpost.in/dipin-damodharan/our-mission-is-todemocra_b_8607538.html

6. Dianne H.B. Welsh and Mariana Dragusin, "The new generation of Massive Open Online Courses (MOOCs) and entrepreneurship education”, Small Business Institute Journal, Vol.9 No.1 pp 51-65, 2013

7. Economic Times [New Delhi]. Retrieved from http://articles.economictimes.indiatimes.com/2014-1209/news/56879752_1_moocsmassive-open-online-courses-coursera.

8. Hattangdi, Ashish, and Atanu Ghosh. "Enhancing the quality and accessibility of higher education through the use of Information and Communication Technologies." International Conference on Emergent Missions, Resources, and the Geographic Locus in Strategy as a part of the 11th Annual Convention of the Strategic Management Forum (SMF), India 2008. Vol. 2011. 2008.

9. MooKIT, http://www.mookit.co/

10. Miller, A. (2017). Sites powered by Open edX , Accessed from : https://github.com/edx/edxplatform/wiki/Sites-powered-by-Open-edX

11. MOOCs Petition, http://www.MOOCsUniversity.org

12. The Manthan Award Digital Annual Festival for Development, tp://manthanaward.org/files/AgendaManthanAwar2012.pdf

13. Pandey, N. (2016, Jul 14). Open online course providers upbeat as enrolments jump. http://www.thehindubusinessline.com/news/education/open-online-course-providers-upbeat-as-enrolmentsjump/article8850328.ece

14. Parag Chatterjee, "Enhancement of digital literacy and excellent education through E-learning for the students in rural parts of West Bengal", 101st Indian Science Congress, Jammu, India, 2014.

15. Shah, D. (2016, Dec 25). By The Numbers: MOOCS in 2016, How has the MOOC space grown this year? Get the facts, figures, and pie charts, https://www.class-central.com/report/mooc-stats-2016.

16. Shah, D. (2016, Jul 8). MOOC Tracker - Never Miss a Course, Notification/Reminder service for MOOCs, Class Central. Accessed from https://www.class-central.com/report/mooc-tracker

17. Wikipedia, http://en.wikipedia.org/wiki/Massive_open_online_course. 